\title{
Prospects and Challenges of Multi-Layer Optical Networks
}

\author{
Ken-ichi SATO $^{\dagger \text { a) }}$, Fellow and Hiroshi HASEGAWA ${ }^{\dagger}$, Member
}

\begin{abstract}
SUMMARY This paper investigates the prospects and challenges of hierarchical optical path networks. The merits and issues of introducing higher order optical paths are elucidated. State of the art of the key enabling technologies are demonstrated including hierarchical optical cross-connect switch architectures, hierarchical optical path network design algorithms, a newly developed waveband filter, and waveband conversion technologies. key words: optical path, waveband, photonic network, multi-layer optical paths, optical cross-connect
\end{abstract}

\section{Introduction}

Broadband access including ADSL and FTTH is being rapidly adopted throughout the world and as a result traffic is continually increasing. In order to cope with this, optical transmission and node technologies are advancing steadily. The maximum number of WDM wavelengths per fiber now exceeds one hundred, and the maximum channel speed of $40 \mathrm{~Gb} / \mathrm{s}$ are now being intruduced. The key enabling technologies that enhance node throughput and cost reduction, recognized to be optical path technologies [1][3], exploit wavelength routing. Wavelength routing using ROADMs (Reconfigurable Optical Add/Drop Multiplexers) has recently been introduced and a large scale deployment is being conducted in North America to develop cost-effective networks [4], [5]. GMPLS (Generalized Multi-Protocol Label Switching) controlled OXCs (Optical Cross-connects) [6] have also been used to create nation-wide testbeds [7][9]. New broadband services including IP TV and high definition TV are now imminent and further traffic expansion is expected in the near future. Cutting-edge applications including e-science, all of which need enormous bandwidth, are also conceived [10], [11]. This will result in a significant increase in the number of wavelength paths that should be cross-connected at nodes, and hence optical node throughput must be enhanced. One important technology that can resolve this problem is the introduction of wavebands and hierarchical optical path cross-connects (HOXCs) [12]-[14]. This paper explores recent advances in the research and development of hierarchical multi-layer photonic networks.

First, we discuss transport network evolution focusing on path technologies. The hierarchical electrical paths

Manuscript received September 20, 2006.

Manuscript revised February 13, 2007.

${ }^{\dagger}$ The authors are with the Department of Electrical Engineering and Computer Science, Nagoya University, Nagoya-shi, 4648603 Japan.

a)E-mail: sato@nuee.nagoya-u.ac.jp DOI: $10.1093 /$ ietcom/e90-b.8.1890 and the cross-connection have been utilized since the PDH (Plesiochronous Digital Hierarchy) era [15], [16]. The digital path concept essentially reached fruition with the invention of SDH (Synchronous Digital Hierarchy) and the hierarchical digital path technology formed the basis of modern telecommunications networks. The optical realization of the path concept utilizing wavelength routing [17]-[19] has been intensively investigated since the early 1990's [1]-[3] and practical implementation [7]-[9] and commercial application started very recently as mentioned above. Some early considerations of the hierarchical optical path were seen in the late 1990's [12]. In Sect. 2, the role of the hierarchical optical path, the next evolution step envisaged for the optical path, is elucidated.

We then discuss network design issues. The network should handle hierarchical optical paths, wavelength paths and wavebands (WBs) that consist of multiple wavelength paths. Several studies have targeted the development of hierarchical optical path network design algorithms [20], [21], and have demonstrated the advantage of introducing HOXCs; most works evaluated the effectiveness in terms of the total number of HOXC ports compared to that of single-layer OXCs. Another study [22], [23] showed that a HOXC with non-uniform WB size can not only improve node throughput but also reduce cross-connect node cost. These design algorithms tend to require a complex procedure for resolving wavelength and the waveband assignment problem when wavelength/waveband conversion is not utilized. Recent algorithms [21] introduce a novel traffic demand expression in a Cartesian product space and are explained here in detail.

Next, we discuss some key hardware technologies that are needed to develop HOXCs. They include hierarchical optical cross-connect architectures that consist of waveband cross-connects (BXCs) and wavelength path crossconnects (WXCs). Few studies have been done so far on these switch architectures. The switch scale necessary for single-layer OXCs and HOXCs are evaluated considering the different levels of switch flexibility that yield graceful expansion. The conditions wherein HOXCs are superior to single layer WXCs are clarified. We then discuss waveband multi/demultiplexers (WB MUX/DEMUX). We describe a newly developed WB MUX/DEMUX [24], [25] that uses two concatenated cyclic AWGs (Arrayed-Waveguide Gratings). The salient feature of the device is that it can accommodate multiple input fibers simultaneously and the port utilization of the device is shown to be $100 \%$ with bi- 


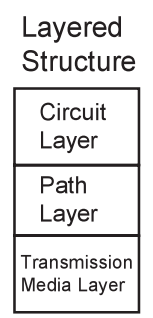

Introduced:

New

Technology

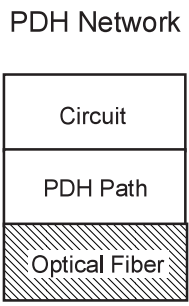

1981
ATM Network

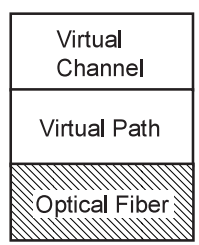

1995

ATM Transport System

Optical Technologies
Photonic Network

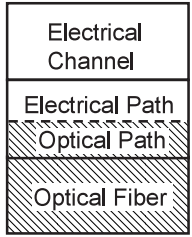

2002

Optical Path

Technologies

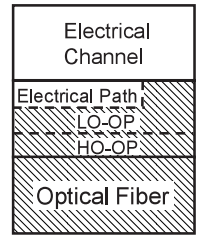

$20^{* *}$

Hierarchical Optical Path Technologies Co-axial Cable System (32 Mb/s) Transmission System
LO-OP: Lower-order Optical Path HO-OP: Higher-order Optical Path

Fig. 1 Transport network technology evolution in Japan.

directional input fibers. We finally discuss recent advances in waveband conversion technologies. The technologies discussed in this paper will need to be consolidated to realize hierarchical optical path networks that offer a solution to the expected traffic increase and the ever increasing power consumption of node systems.

\section{Hierarchical Path Network Architectures}

\subsection{Role of Path and Hierarchical Electrical Path Archi- tectures}

A layered transport network architecture has been developed that offers simplified network design, development, and operation, and offers smooth network evolution [26]. It also makes it easy for each network layer to evolve independently of the others by capitalizing on the introduction of new technologies specific to each layer. The layering concept has been extensively discussed within ITU-T for the SDH transport network [27], [28]. Traditionally, the network can be divided into three layers from the viewpoint of functions: a physical media layer, a transmission path layer, and a communication circuit (/flow) layer. The circuit/flow provides end-to-end communication dynamically or on the basis of short term provisioning. The circuit/flow layers are dedicated to specific services. The transmission media, which physically interconnect nodes and/or subscribers, is constructed based on long-term provisioning; geographical conditions are taken into consideration. The path layer bridges these two layers and plays an important role in constructing reliable and flexible networks [26]. Important attributes of a path are; a grouped circuit/flow serving as a unit of network operation (including traffic engineering), design and provisioning, and an object to be manipulated for recovering from node and transmission line/system failures. Network flexibility can also be enhanced with path layer control. Path layer functions can be materialized with intelligent ADM (Add/Drop Multiplexer) systems and/or digital cross-connect systems and control systems.

Different paths have been introduced in accordance
Table 1 Multiplexing and path realization technologies.

\begin{tabular}{|c|c|c|c|c|}
\hline \multirow{2}{*}{$\begin{array}{l}\text { Multiplexing } \\
\text { Technologies }\end{array}$} & \multicolumn{4}{|c|}{ Path Technologies } \\
\hline & Path & $\begin{array}{c}\text { Identification } \\
\text { (\# of Paths/Link) }\end{array}$ & $\begin{array}{l}\text { Soft/Hard } \\
\text { State }\end{array}$ & Routing \\
\hline $\begin{array}{l}\text { PDH } \\
\text { SDH }\end{array}$ & $\begin{array}{c}\text { Digital Path } \\
\text { (SDH: VC-1/2, } \\
\text { VC-3/4) }\end{array}$ & $\begin{array}{l}\text { Time Position in } \\
\text { the TDM Frame } \\
\qquad(<768)\end{array}$ & Hard & $\begin{array}{c}\text { Time Slot } \\
\text { Interchange } \\
+ \\
\text { Space Switch }\end{array}$ \\
\hline ATM & VP & $\begin{array}{l}\text { Cell Header (VPI) } \\
\quad(<4096 ; \text { NNI } \\
\quad<128 ; \text { UNI })\end{array}$ & Soft & $\begin{array}{c}\text { Store-\&-Forward } \\
\text { Electrical Processing } \\
+ \\
\text { Space Switch }\end{array}$ \\
\hline Packet & LSP & $\begin{array}{l}\text { Shim Label } \\
\qquad\left(<2^{16}\right)\end{array}$ & Soft & $\begin{array}{c}\text { Store-\&-Forward } \\
\text { Electrical Processing } \\
+ \\
\text { Space Switch }\end{array}$ \\
\hline WDM & Optical Path & $\begin{array}{c}\text { Wavelength } \\
(<1,000) \\
\text { Waveband }(<100)\end{array}$ & Hard & $\begin{array}{c}\text { Waveguide Router } \\
\text { (Self-Routing) } \\
\text { and/or } \\
\text { Space Switch }\end{array}$ \\
\hline
\end{tabular}

with the evolution of transfer techniques; digital paths (virtual containers) in SDH [29], Virtual Paths in ATM (Asynchronous Transfer Mode) [30]-[32], Label Switched Paths in IP/MPLS (Multi-Protocol Label Switching [33], [34]), and optical paths for photonic networks [1]-[3]. The Japanese example of path technology evolution is depicted in Fig. 1. The characteristics of different paths are summarized in Table 1. GMPLS [35], [36] has been proposed as a control technique. It aims at providing a common control mechanism among different path layers (and fiber layer) that utilizes IP-based signaling; intensive technical development and standardization activities have been undertaken.

\subsubsection{Hierarchical Path Arrangement}

Hierarchical path arrangements have been developed and widely utilized. One typical example is a hierarchical SDH digital path network, which is shown in Fig. 2 [27]. In circuit switching, connection admission control is done on the basis of the usage of paths that have been pre-established between switches to meet traffic demands. The role of the path is called service-access. In SDH networks, the service-access path bandwidth is $1.5 \mathrm{Mb} / \mathrm{s}(24 \times 64 \mathrm{~kb} / \mathrm{s}$ channels; Japan and North America) or $2.0 \mathrm{Mb} / \mathrm{s}(32 \times 64 \mathrm{~kb} / \mathrm{s}$ channels; Europe) for telephone service. Transmission system bit-rates, 


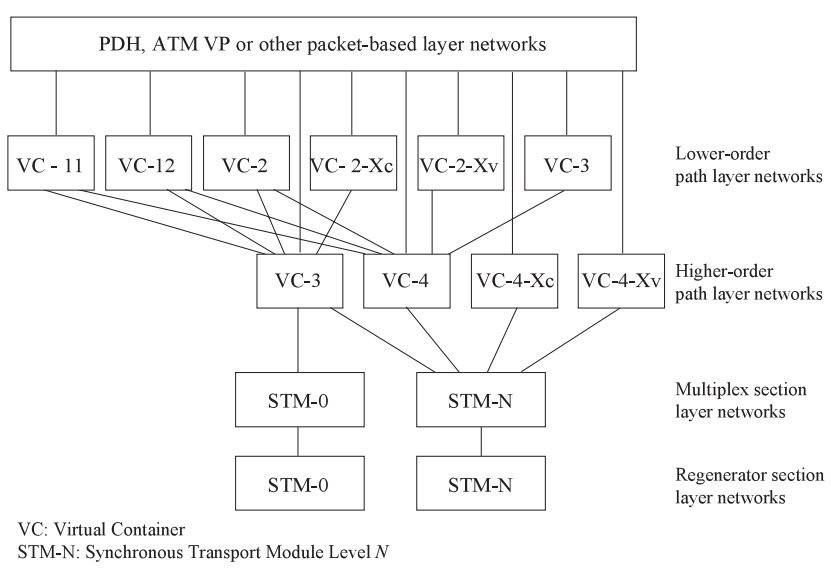

Fig. 2 Hierarchical path layer structure in SDH.

however, are much larger than the service-access path bandwidths and now reach forty giga bits per second. Traffic demands between any two nodes are not always sufficient to fill the bandwidth. This has triggered the use of another path stage, higher order paths ( 49 or $150 \mathrm{Mb} / \mathrm{s}$ ), to accommodate several service-access paths. The higher order paths are accommodated within a higher capacity transmission link and are cross-connected [16], [37] at transit nodes to construct an economical transport network. The role of the higher order paths is, therefore, called trans-access. The higher order paths can be used to separate IP networks and traditional telephone and data networks and to share a physical media network [38] among the services. The higher order paths are usually used for network protection/restoration against failures. The classification of service-access and trans-access is not strict, since higher order paths can be directly used to provide large bandwidth services such as video and leased line services. The hierarchical bandwidth paths are thus very useful for creating an efficient network and hence are widely utilized in the SDH network. On the other hand, the hierarchical path structure and the multiple stages of the multi/demultiplexing, can complicate path accommodation designs and may lead to poor link utilization. For example, if the multiplexing efficiency of the lower stage path into the next stage is 0.8 for each multiplexing stage, three stage multiplexing results in the final link utilization of 0.5 $\left(=0.8^{3}\right)$. This inefficiency can be removed with the logical realization of paths such as VPs in ATM and LSPs in IP/MPLS. In LSP, a shim header is defined to be hierarchical at the initial standardization stage [33] so that capabilities of LSPs can be enhanced as needed.

\subsection{Optical Path and the Hierarchical Architectures}

Optical paths provide for the wavelength routing of signals, which eliminates rather expensive OE/EO (Optical to Electrical/Electrical to Optical) conversion, enables bit rate and transfer mode (protocol) independent cross-connects, dramatically enhances node throughput, and greatly reduces node system power consumption. These are discussed in
Present Network

Future Network

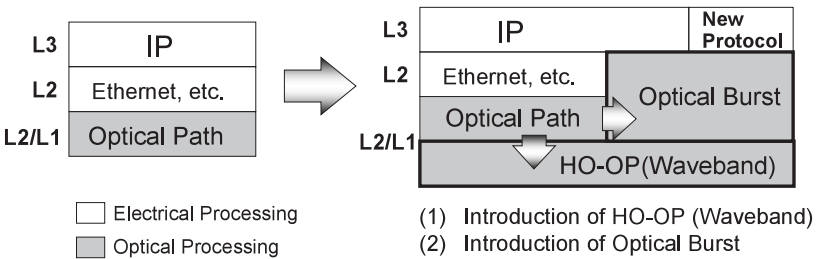

Fig.3 Different paths to network throughput expansion.

detail in [26]. Optical paths are first utilized as trans-access paths since the bandwidths are usually higher than $2.5 \mathrm{~Gb} / \mathrm{s}$; they accommodate electrical lower order paths such as SDH digital paths, and LSPs.

Routing functions in the optical domain with optical paths were first put into commercial use with the employment of ROADMs. OXCs have been used for creating nation-wide testbed networks. With regard to optical technologies, however, large scale optical switch technologies are not mature and the restrictions are still substantial. When the large scale deployment of OXCs will start depends on the degree of both the traffic and switch technology maturity. The OXCs will be deployed to interconnect multiple ROADM rings or to create mesh-based networks.

One of the salient features of optical paths is that switch complexity does not depend on the bit rate carried by an optical path. With electrical technologies, switching becomes more and more difficult and consumes more electrical power as the bit rate increases. Thus, the wide deployment of optical path technologies will be driven by the traffic increase, and the limit in terms of power consumption and throughput inherent in electrical switching. With regard to the node throughput enhancement, different directions have been explored as shown in Fig. 3 [39]; introduction of higher-order optical paths (wavebands) and the introduction of optical burst switching. It will take much longer for the latter to yield a practical application than the former, and so only the former is discussed below.

Hierarchical optical path arrangement is a natural approach when traffic increases, and when optical layer services such as OVPN (Optical Virtual Private Network) services, lambda leased line services, and lambda switched services for e-science emerge. The optical paths are used as a service-access path, and the introduction of higher-order optical paths is envisaged. Another important point is that some optical switches support optical signals with a wide range of wavelengths; this means that the same switches can be used for switching multiple optical paths. Switching multiple optical paths or switching wavebands can reduce total switch size (necessary number of cross-connect switch ports) substantially. This mitigates one of the major present challenges in creating large scale optical crossconnects. The hierarchical path structure can degrade link utilization, and this effect may be enhanced by the additional complexity created by the waveband/wavelength assignment problem. This problem can be mitigated by the 
development of effective hierarchical optical path network design algorithms. They minimize total network cost that consists of node and link cost, or total number of optical cross-connect ports. These merits and demerits are evaluated in the following sections.

\section{Hierarchical Photonic Network Enabling Technolo- gies}

In order to create hierarchical photonic networks that warrant introduction, substantial technology development is still necessary. Figure 4 depicts some of the key issues that need further R\&D and are discussed in the following sections. Recent development and future challenges are described.

\subsection{Hierarchical Optical Path Network Design}

The waveband routing and waveband assignment problem of multi-granular optical networks is a generalization of the single-granular optical network design problem. The problem aims at minimizing cost functions subject to waveband and wavelength continuity constraint. Analogous to the single-granular case [40], the problem is inherently NPcomplete and can equivalently be formulated as a combinatorial optimization problem that targets minimizing the total number of optical ports [41]-[43] of cross-connect systems or maximizing the utilization of fiber capacity [44]. The number of binary variables in the combinatorial optimization problem explosively increases with network size. This characteristic makes the problem computationally impossible to accurately solve for large networks. Indeed, previous publications that tackled the combinatorial optimization formulation gave up on exact solutions and provided alternative algorithms based on heuristics or relaxation instead. Several such methods have been proposed [41]-[47]; they are categorized as follows.

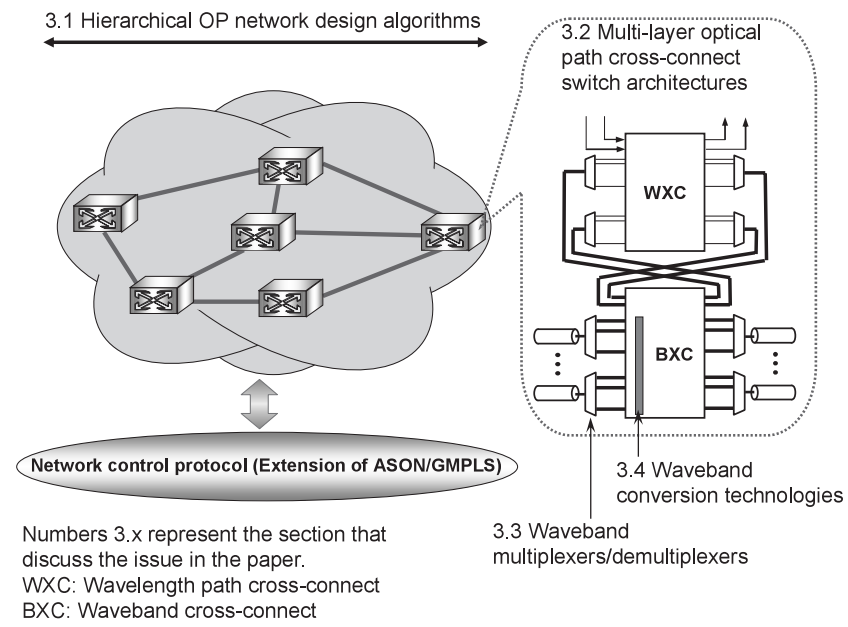

Fig. 4 Key technologies necessary to develop hierarchical optical path networks.
3.1.1 Grooming of Wavelength Paths Having Common Source/Destination or Partially Shared Routes [46], [47]

Wavelength paths with the same destination are first collected and then accommodated within wavebands [46]. This approach can also be applied to wavelength paths having the same source. Since the requirement of having the same destination is rather restrictive, an alternative [47] computes the routes of all wavelength paths first and then locates sets of wavelength paths that share common routes at least for some significant distance. Wavebands are then constructed along these shared routes. This kind of method is simple because it is almost equivalent to a design method for single-layer optical path networks. However, further aggressive grooming is necessary to improve waveband utilization ratio especially when traffic demand is not large.

\subsubsection{Waveband Tunnel Construction First [45]}

The potential traffic loads of all fibers are estimated first and the amounts of potential incoming and outgoing traffic of each node are defined by using the potential loads. After connecting pairs of nodes that have large incoming and outgoing traffic loads by waveband tunnels, wavelength paths are accommodated within the waveband tunnels based on the shortest paths. Reference [45] focuses on dynamic wavelength paths allocation that is necessary in realizing optical circuit-switch type services.

\subsubsection{On Demand Waveband Assignment [42], [43]}

This approach assigns the routes of wavelength paths one by one. For each route assignment, the optimal route is identified considering existing waveband or fiber tunnels. If no proper tunnel is available, new tunnels are constructed. The approach tries to find only a shortest path at each wavelength path accommodation. The waveband tunnels will be satisfactory filled whereas it lacks global optimization of waveband tunnel placement.

\subsubsection{Relaxation-Based Methods [41], [44]}

One approach [41] divides the original combinatorial optimization problem into three easier sub-problems that consist of other combinatorial optimization problems. An alternative approach [44] makes the original problem easier by using Lagrangean Relaxation. Both methods then resolve the easier problems so as to derive an approximation of the optimal solution. This kind of approach is useful mainly for small networks where the sub-optimal solutions can be derived with a slight degree of relaxations.

\subsubsection{Cluster-Search Method in a Source-Destination Cartesian Product Space [21], [48]}

Except for the relaxation-based method, the aim of the 


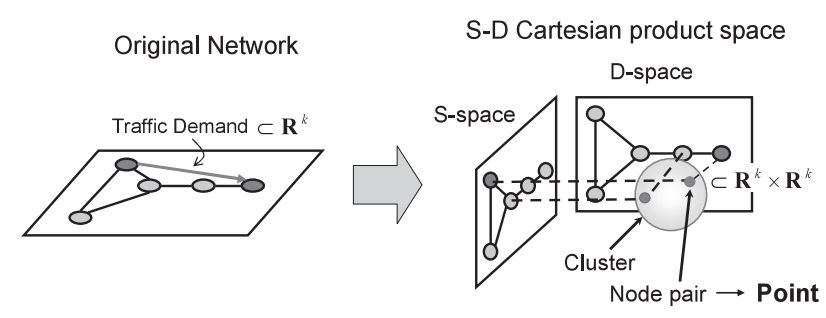

> A Cartesian product space is introduced, in which a node pair is represented as a point, and the traffic demand is represented as the degree of shading or size of the point, etc.

$>$ Nearby traffic demands are classfied as clusters of points.

$>$ The proposed method searches for clusters in the space and assigns them to wavebands.

Fig. 5 Traffic demand and clusters in S-D Cartesian product space.

above heuristics is to improve utilization of waveband paths as well as to reduce node cost incurred by carrying each wavelength path by minimizing wavelength path crossconnection at intermediate nodes. Suppose that we have waveband paths between every node pairs where traffic demand exist, then the node cost of carrying each wavelength is generally reduced, however, waveband utilization may be degraded.

A heuristic algorithm that is based on a different strategy was recently proposed [21], details and a performance analysis are given in [48]. To minimize the total cost, which consists of node cost and link cost, the wavelength path cross-connection at intermediate nodes must be reduced and at the same time waveband utilization must be maximized. We must therefore search for a set of wavelength paths that are efficiently carried by a waveband path and can sufficiently occupy the waveband path. A space, named "s-d (source-destination) Cartesian product space," is newly defined to evaluate closeness among wavelength paths. The space can be used to effectively search for waveband paths that can reduce total cost (See Fig. 5). For each set of wavelength paths, a waveband path is constructed so as to maximize the degree of cost reduction. The wavelength paths that are not accommodated with the first step are finally accommodated in wavebands by identifying the shortest paths in a multi-layered graph considering waveband tunnels (See Fig. 6).

The multi-layer optical network can reduce the number of optical ports, but will lower fiber utilization levels. It is therefore important to clarify the conditions under which the waveband approach is attractive. Figure 7 shows the cost reduction possible with the multi-layer optical path network in a comparison against a single layer one for a $9 \times 9$ polygrid network with randomly distributed wavelength path demands. The vertical axis of the graph is relative cost normalized by the cost of a single layer optical network designed by locating the shortest paths with re-routing of wavelength paths in sparsely used fibers. End-to-End represents the method that accommodates wavelength paths within a waveband path that has the same source and destination as the wavelength paths. Even this simple scheme, End-to-End, achieves approximately $40 \%$ cost reduction when the average wavelength path demand between nodes equals wave-

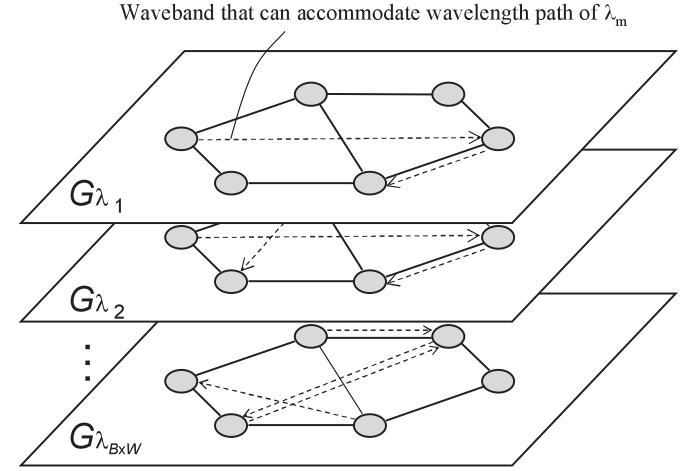

Fig. 6 Multi-layered graph where each layer is assigned to each wavelength.

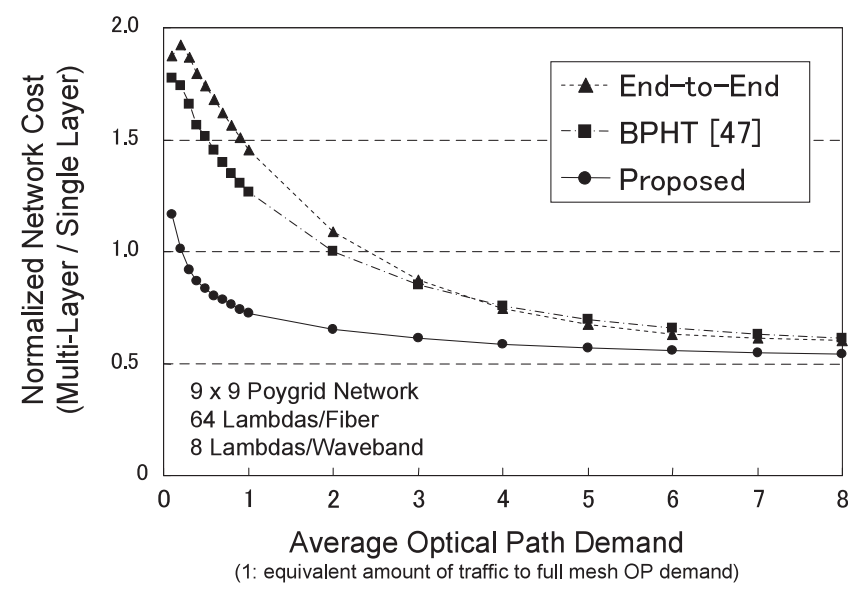

Fig. 7 Comparison among proposed and conventional algorithms (endto-end and BPHT).

band width. With the method in [21], [48], the cost reduction is greatly enhanced even for smaller traffic demands. The investigation provides not only performance comparisons of different methods, but also clarifies the applicability of the multi-layer optical path network.

\subsection{Multi-Layer Optical Path Cross-Connect Switch Ar- chitectures}

\subsubsection{Basic Cross-Connect Node Architecture}

In a single-layer $\mathrm{OXC}$, wavelength paths are de-multiplexed by DEMUX, routed by WXC (Wavelength path XC), and then recombined by MUX (See Fig. 8(a)). Wavelength converters can be inserted before or after WXC so as to avoid wavelength collision. The number of wavelength converters may be limited because of their cost; collision can be reduced by applying effective routing and wavelength assignment algorithms [49]-[52].

The switch architectures for multi-layer optical path networks are determined to suit the multi-layer optical path arrangements. Figure 8(b) depicts a generic configuration of the hierarchical optical cross-connect switch architecture. HOXC is divided into two parts as shown in Fig. 8(b). One 


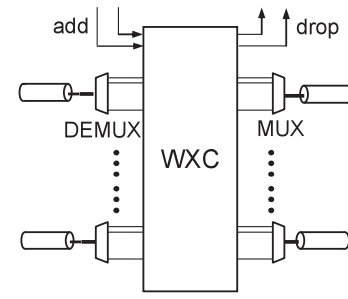

(a) Single layer switch architecture.

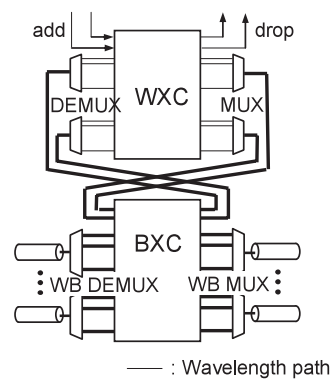

(b) Hierarchical switch architecture.

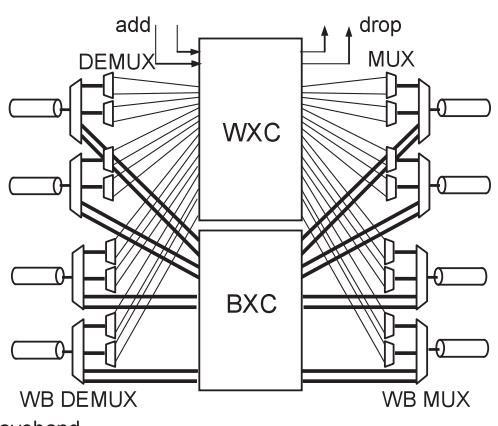

(c) Non-hierarchical switch architecture.

Fig. 8 Different cross-connect switch architectures

part consists of WB MUX/DEMUX and BXC (waveband $\mathrm{XC}$ ) for routing higher-order waveband paths, and the other part consists of MUX/DEMUX and WXC for routing lowerorder wavelength paths. The routing of optical paths should be processed by $\mathrm{BXC}$ as much as possible so as to make the best use of the HOXC arrangement. In other words, the WXC part should only be used when WB routing is not possible. This is possible due the network design algorithms, which accommodate wavelength paths into wavebands and wavebands into fibers as described in the previous section.

Several studies [53] proposed non-hierarchical OXCs (see Fig. 8(c)) where wavelength paths and WBs are accommodated simultaneously in a fiber. At a node, wavelength paths and WBs are fed to a WXC and a $\mathrm{BXC}$, respectively. With this arrangement, they are treated independently. Grooming of wavelength paths at the intermediate OXCs are not done and as a result no interworking between wavelength path cross-connects and waveband crossconnects is required, which simplifies the switch architectures. This arrangement can decrease the number of the total cross-connect switch ports needed when the number of optical paths per waveband is within a certain range [53], however, if the traffic pattern or traffic volume changes, the optimum wavelength path and waveband configuration changes and as a result, the effectiveness decreases. Furthermore, the flexibility of the cross-connect switch is very limited since the ratio of the number of wavelength paths to that of WBs accommodated within a fiber has to be fixed to optimize the switch architecture. We, therefore, do not discuss this approach below.

Different switch architectures and technologies have been utilized to create optical switches. They include 2dimensional [54] and 3-dimensional (3D) MEMS [55], a PLC (Planar Lightwave Circuit) [56] switch, and a mechanical fiber switch. 3D MEMS switches are attractive due to their high functionality and low optical insertion loss and crosstalk [57]. The WSS (Wavelength Selective Switch) [58], [59], which was originally proposed as the Delivery and Coupling Switch (DC-SW) [60]-[62] in 1993 as described below, uses 3D MEMS switches, and is going to be utilized to develop ROADMs for applications in metropolitan area networks. Since the micro mirrors must be controlled in three dimensions and require extreme assembly and operation precision; reliability of large scale 3D MEMS switches in various conditions must be verified in more detail. On the other hand, 2D MEMS and PLC switches (two dimensional matrix-type switches) are attractive due to their proven reliability [63] and high manufacturing yields. This paper, therefore, focuses on $\mathrm{BXC}$ and $\mathrm{WXC}$ architectures assuming the use of the latter switch technologies.

\subsubsection{Flexibility for Expansion}

To realize the economical introduction of optical crossconnects and subsequent expansion to cope with traffic growth, the system must offer modular growth capability. The following attributes of expansion are important and are explained in Fig. 9.

- modular growth regarding fiber

The number of input/output fiber ports is incrementally increased as traffic demand increases. This minimizes the initial investment with regard to fiber ports.

- modular growth regarding WB

The number of WBs is incrementally increased as traffic demand increases. This minimizes initial investment with regard to the ports connecting WB MUX/DEMUX and BXC. - modular growth regarding wavelength paths in WB The number of wavelength paths in WB is incrementally increased as traffic demand increases. This minimizes initial investment with regard to WXC.

- modular growth regarding add/drop WB

The number of added WBs from WXC to BXC or dropped $\mathrm{WBs}$ from BXC to WXC is incrementally increased as traffic demand increases. This minimizes initial investment with regard to the ports connecting $\mathrm{BXC}$ and $\mathrm{WXC}$.

In the following discussion, $N$ is the number of input/output fibers and $m$ is the number of wavelength paths per fiber. Variable $x$ is introduced to stand for the ratio of wavelength paths that are to be added/dropped for connections to electrical systems (digital cross-connect systems or routers, etc.) at a node.

The first flexibility can be realized by using the Delivery and Coupling Switch (DC-SW) [60]-[62]. One example for the single layer OXC is shown in Fig. 10(a). This OXC utilizes $N, m(1+x) \times N(1+x)$ DC-SWs and one switch is dedicated to each input fiber. If this architecture is used for 


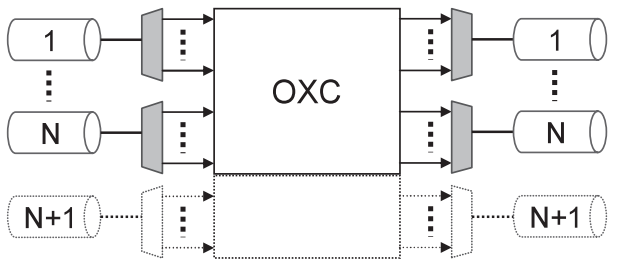

(a) Modular growth: fiber.

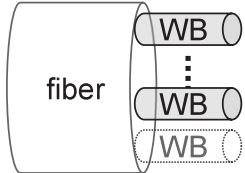

(b) Modular growth: waveband.

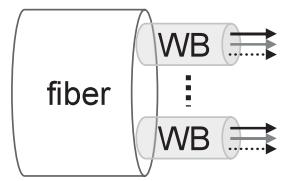

(c) Modular growth: wavelength path.

Fig. 9 Different levels of flexibility.

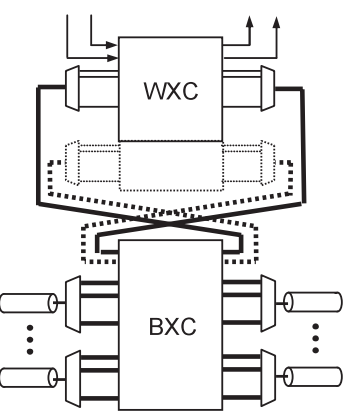

(d) Modular growth: add/drop waveband.

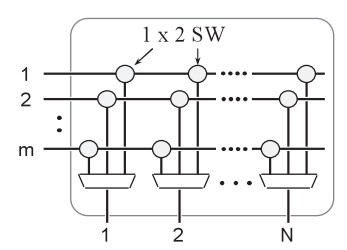

(b) Matrix type $m \times N$ DC-SW.

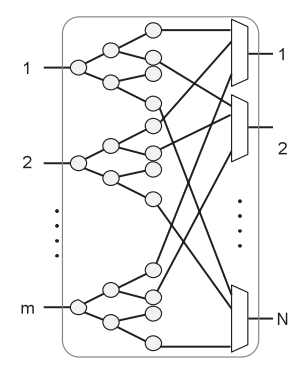

(c) Tree type $m \times N$ DC-SW.

(a) Single layer OXC architecture that uses DC-SWs.

Fig. 10 Single layer OXC using Delivery and Coupling switches.

BXC in HOXC, then the MUX in the figure should be WB MUX. A matrix type arrangement of the $m \times N$ DC-SW is shown in Fig. 10(b) and a tree type arrangement is shown in Fig. 10(c). This DC-SW configuration was originally proposed in 1993 [60]-[62] and has been used in the optical cross-connect systems used to create nation-wide testbed networks [7]. The DC-SW is now often called the WSS (Wavelength Selective Switch). In Figs. 10(b) and 10(c), $N$ MUXs are used when the wavelength of each input signal is fixed, however, an optical star coupler can be used instead of the MUX when the wavelength of each input signal can change. The DC-SW allows any of the $m$ incoming optical signals to be connected to any of the $N$ outgoing ports. These outgoing port signals are coupled to output fiber ports by using a star coupler (SC). DC-SW is composed of $1 \times 2$ optical SW elements. This architecture makes the value of $x$ (Fig. 10(a)) equal among different fibers. The configuration provides modular growth capability in terms of input and output fiber pairs [26], [60].

The second flexibility can be realized by using a singlelayer OXC that has a $N(1+x) \times N(1+x)$ matrix SW for each optical path group with the same wavelength as shown in Fig. 11. This architecture makes the value of $x$ equal among different wavelengths. The number of matrix SWs, $m$, equals the number of wavelength paths per fiber. Each $N(1+x) \times N(1+x)$ SW assigns an output fiber port to each wavelength path. This configuration has modularity in terms of wavelength path. HOXC architectures can re-

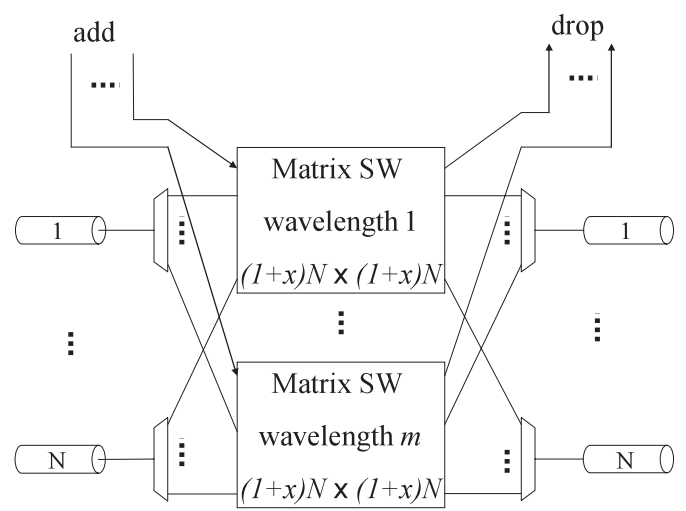

Fig. 11 Single layer OXC that consists of matrix switches dedicated to the same wavelength paths.

alize this flexibility when the BXC utilizes the above type of matrix switch for each waveband group in combination with a WXC that has modular growth capability in terms of wavebands.

The third flexibility is realized in the most simple case at the sender and receiver side; corresponding transceivers and receivers need to be added, which will be almost the same procedure as the wavelength increase process for WDM transmission channel increase. In regard to the switch, if the switch architecture uses switches, each of which correspond to a wavelength path group with the same wavelength, then the addition of a new wavelength path 


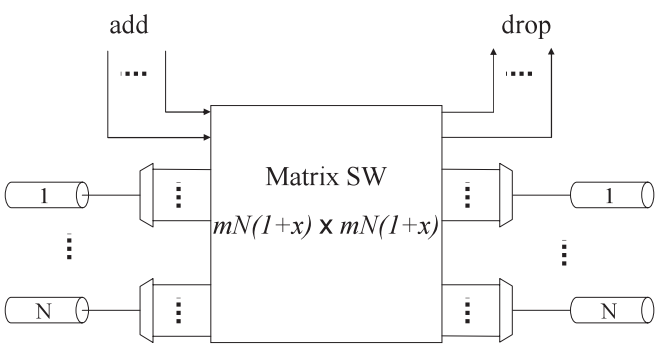

Fig. 12 Single layer OXC with a large matrix switch.

group will allow modular growth of the switch.

The fourth flexibility is realized in HOXC when WXC has modular growth capability in terms of wavebands.

\subsubsection{Single-Layer OXC SW Architecture}

First, we briefly discuss single-layer OXC switch (SW) architectures. The simplest switch architecture for a singlelayer $\mathrm{OXC}$ is one that uses large $m N(1+x) \times m N(1+x)$ matrix switches as shown in Fig. 12 or that uses the equivalent Clos switching network [64]. It is possible to route an optical path from any input port to any output port. The configuration does not possess any of the flexibilities described above.

For the single-layer OXC with large matrix switch, the switch scale is about ten times bigger than that of any other SW architecture considered here. DC-SW is the best in terms of SW size, however, it requires many more MUXs/DEMUXs than the other architectures; the number is proportional to order $N^{2}$. The $\mathrm{SW}$ architecture requires $N$ SCs which leads to $10 \times \log N \mathrm{~dB}$ intrinsic loss, which may be an issue when $N$ becomes large. The single-layer OXC that consists of matrix SWs dedicated to the samewavelength paths, shown in Fig. 11, can be constructed with relatively small scale switches and the necessary number of MUXs/DEMUXs is low. As a result, the SW architecture based on matrix SWs for each same-wavelength path group is attractive for constructing single-layer OXCs with regard to cost and loss, if modularity in terms of input/output fibers is not necessary.

\subsubsection{HOXC SW Architectures}

Different HOXC switch architectures have been developed and compared in terms of cost, loss and flexibility [65]. The WXC SW architecture adopted for developing HOXC, shown in Fig. 11, is attractive for single-layer OXCs in terms of switch scale and MUX requirement, as discussed above. Let $N$ be the number of fibers, $m$ that of wavelength paths per fiber, $k$ that of WBs per fiber, and $l$ that of wavelength paths per WB. Variable $y$ represents the ratio of WBs dropped at a node to total WBs delivered to the node (or the ratio of WBs added at a node to total WBs launched from the node). Thus, the product of $k$ and $l$ equals $m$, and $y$ ranges from 0 to 1 . Among switches compared, the most attractive switch architecture for $\mathrm{BXC}$ is the one shown in Fig. 11 for the WXC case. The BXC consists of $(1+y) N \times(1+y) N$ matrix SWs for each WB. The architecture makes the value of $y$ equal among the different WB groups. Each $(1+y) N \times(1+y) N \mathrm{SW}$ assigns an output port to each WB. This configuration has modular growth capability in terms of WBs, and can reduce WXC switch size by setting the proper limit $(y)$ to the number of add/drop WBs. Namely, this architecture realizes the fourth flexibility described above, modular growth capability in terms of add/drop WBs. This architecture, therefore, demonstrates very attractive characteristics for developing BXCs in terms of cost, loss, and flexibility [65].

As discussed for the single-layer OXC, the DC-SW offers the smallest switch scale, but the number of $\mathrm{WB}$ MUXs/DEMUXs needed increases with the second order of power $N$. This architecture will be cost-effective when the large scale integration of WB MUXs becomes possible and a substantial cost reduction is attained.

\subsubsection{Comparison of Switch Scale between Single-Layer $\mathrm{OXC}$ and $\mathrm{HOXC}$}

For OXCs, some of the input and output ports of a switch are used for intra-office interconnections between optical switches and electrical systems such as electrical digital cross-connect systems and routers. To minimize total optical switch size, it is essential to realize add/drop ports in an optimal manner. There are two ways to realize add/drop ports [65]. One utilizes a part of the matrix SW ports as add/drop ports. Because this system can restrict the number of add/drop ports to those needed, this system can minimize the number of add/drop ports. However, this system increases matrix $\mathrm{SW}$ size. The other realizes add/drop ports based on the use of $2 \times 2$ SWs.

A $2 \times 2 \mathrm{SW}$ is added to each output port of the matrix SW. With this arrangement, the matrix SW size does not depend on the existence of add/drop ports, that is, this system can minimize the matrix SW size. However, this arrangement cannot restrict the number of add/drop ports and allows all input signals to the matrix switch to be dropped.

Here, the ratio of add/drop wavelength paths (between optical switches and electrical systems) to all wavelength paths delivered to the node is denoted as $x$ as explained before. For HOXCs, the add/drop wavelength paths between optical switches and electrical systems are restricted through two stages of switches, BXC and WXC. BXC restricts the ratio to $y$, as described before, and then $\mathrm{WXC}$ restricts it to $x$, which results in the ratio of $x y$. In the following evaluations for HOXCs, $x$ is set to 1 , in other words, the restriction is done only in the BXC stage. Comparisons of switch scale between single-layer OXC and HOXC have been done [65] with the condition that $x$ for single-layer OXCs and $y$ for HOXCs were equal. Figure 13 shows evaluated switch size for single-layer $\mathrm{OXC}$ and $\mathrm{HOXC}$, with the two variants defined as using and not using $2 \times 2 \mathrm{SWs}$. In Fig. 13, $N$ is set at $8, m$ is set at 96 , and $k$ is set at 8 . In the single-layer OXC that uses $2 \times 2 \mathrm{SWs}$, the number of add/drop ports can not be 


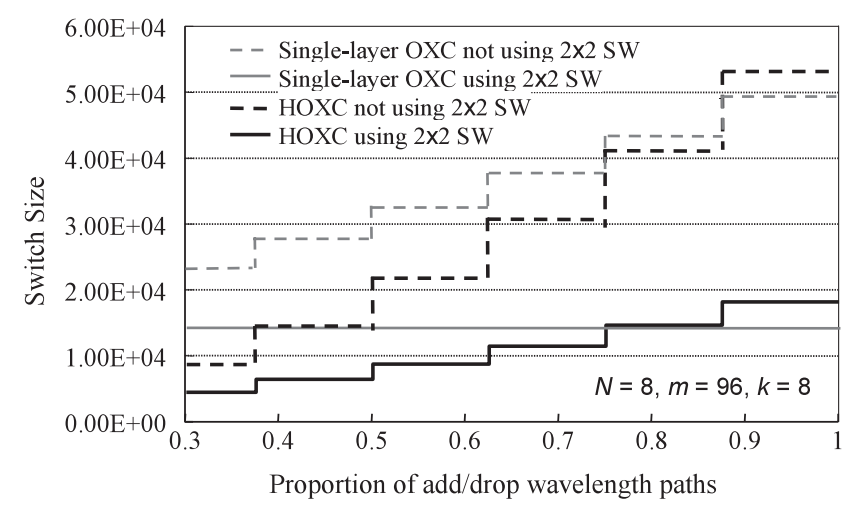

Fig. 13 Comparisons of switch scale between single-layer OXC and HOXC.

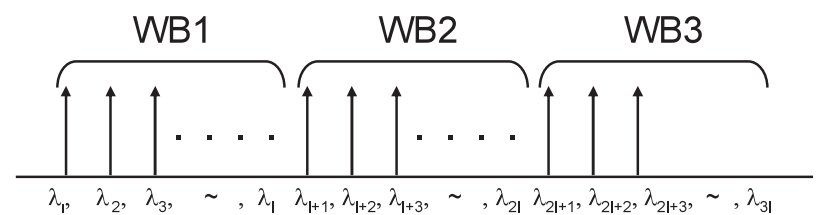

(a) Continuous WB arrangement.

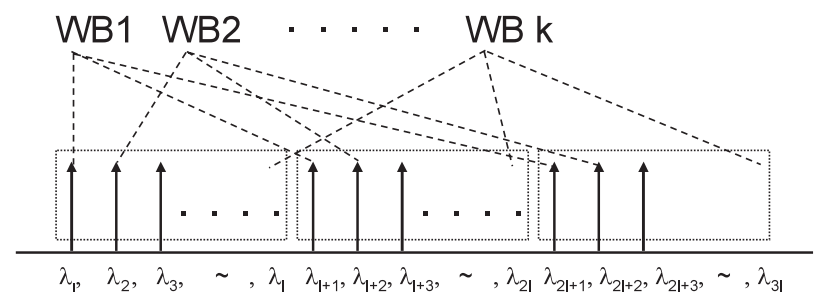

(b) Discontinuous (Interleaved) WB arrangement.

Fig. 14 WB arrangement.

restricted and is constant, $m N$, regardless of $x$. By using $2 \times 2$ SWs, switch size can be greatly reduced in both the singlelayer OXC and the HOXC. This advantage becomes more prominent as $x(y)$ becomes smaller. Figure 13 elucidates the region and the conditions in which HOXCs are more effective. When $x(y)$ is less than 0.5 , HOXC switch size is at least $55 \%$ smaller than that of the single-layer OXC.

\subsection{Waveband Multiplexers/Demultiplexers}

Waveband multiplexers/demultiplexers (WB MUX/ DEMUX) are one of the other key components in developing HOXCs. We first describe different WB arrangements for the hierarchical optical path network. The first one, a conventional arrangement, is the continuous wavelength path arrangement shown in Fig. 14(a). The other, newly proposed in [24], offers the discontinuous (interleaved) wavelength path arrangement depicted in Fig. 14(b). From the networking point of view, the WB arrangement has virtually no effect on network provisioning or OA\&M (Operation, Administration and Maintenance).

WB MUX/DEMUX can be realized in different ways. A thin-film filter has been reported that offers 8 -skip- 0 band operation supporting a total of 32 channels with $100-\mathrm{GHz}$

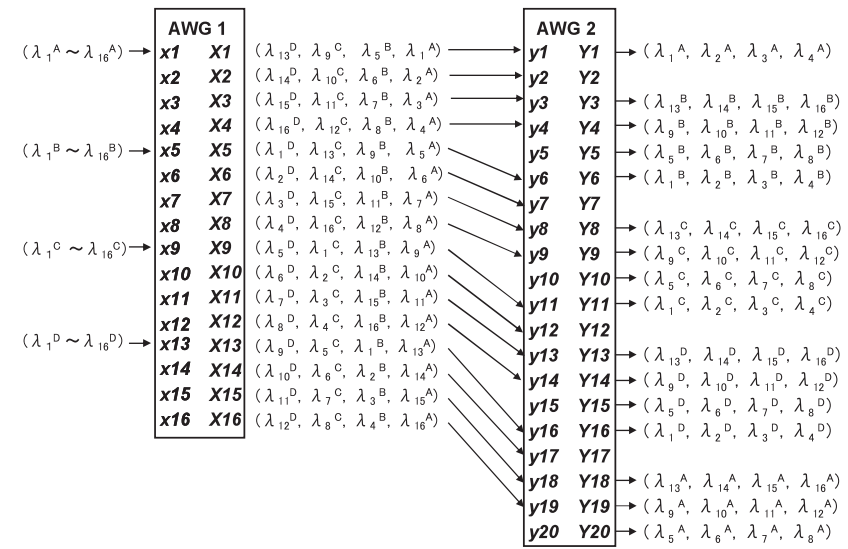

Fig. 15 An example of WB MUX/DEMUX with continuous waveband arrangement.

spacing [66]. Since it requires 409 dielectric thin-film layers [66], the manufacturing challenge is significant. Furthermore, it demonstrates strong non-linear dispersion at the band edges. Other band-filters based on arrayed waveguide gratings (AWG) have been reported. They realize a 17-skip-3 band with a total of 100 channels and 100$\mathrm{GHz}$ spacing [67] and an 8-skip-0 band with a total of 40 channels and 100-GHz spacing [68]. The AWG band filters are susceptible to manufacturing errors that yield adjacent crosstalk. A more recent proposal [24] utilizes two concatenated cyclic AWGs. The point of the novel WB MUX/DEMUX is that it retains multi/demultiplexing granularity at the individual wavelength channel level while outputting the WBs at different ports. This means that conventional cyclic AWGs can be used. The salient feature of the proposed WB MUX/DEMUX is that it can accommodate multiple input fibers simultaneously and demultiplex each band to different output fibers. Two arrangements of paired AWGs have been identified. One eliminates crossings of any waveguides that connect the two AWGs, which can be realized with a continuous waveband arrangement. The other arrangement requires the waveguide crossings, however, it can reduce band crosstalk and maximize output port utilization efficiency; it can be realized using the discontinuous waveband arrangement [24].

Figure 15 depicts an example of the WB MUX/ DEMUX with continuous waveband arrangement [24]; it supports a total of $32100-\mathrm{GHz}$ spaced channels in eight bands. The WB MUX/DEMUX consists of two cyclic AWGs [69], $16 \times 16$ and $20 \times 20$, and waveguides connecting them. The device can accommodate four input fibers (A-D) simultaneously, each of which carries 16 wavelength channels. The device demultiplexes each band on each input fiber and outputs each band separately from 16 (4 bands per fiber $\times 4$ input fibers) output ports out of the 20 ports. This is made possible by the cyclic nature [69] of the AWGs; the wavelength $\lambda_{i}$ at input port \#a $(a=1 \sim N)$ is output from output port $\# b(b=1 \sim N)$ when $i=(a+b-2) \bmod N+1$. Please note that this specific connection arrangement pre- 


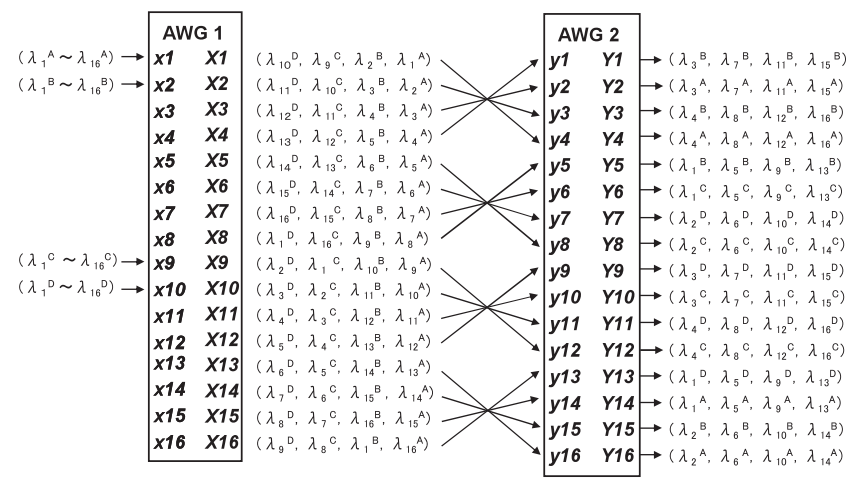

Fig. 16 An example of WB MUX/DEMUX with discontinuous WB arrangement.

vents waveguide crossing, which is advantageous to the monolithic realization of the device. The device feature is generalized as follows. If the number of channels is $m$, and $m=(\#$ of bands per fiber, $k) \times(\#$ of channels in each band, $j$ ), then AWG 1 is $m \times m$ and AWG 2 is $(m+k) \times(m+k)$. This device can support $\min \{m / k, m / j\}$ input fibers simultaneously. The detailed formulation regarding the configurations of the component AWGs and the connection patterns that create WB MUX/DEMUX and prevent waveguide crossings are analyzed in [25].

In the discontinuous WB arrangement, the WB MUX/DEMUX is realized with two cyclic $m \times m$ AWGs. This WB MUX/DEMUX can accommodate $m / k$ input fibers in one device. Figure 16 depicts an example of the WB MUX/DEMUX with the discontinuous waveband arrangement, when $m=16$ and $k=4$. The WB MUX/DEMUX consists of two cyclic $16 \times 16$ AWGs, and waveguides connecting them. This WB MUX/DEMUX has two major differences from the continuous WB arrangement. One is that no connection patterns between AWG 1 and AWG 2 are possible that avoid crossings. The other is that this discontinuous WB arrangement can enhance AWG port usage. Furthermore, this MUX/DEMUX permits the bi-directional connection of multiple input fibers. This can maximize input and output port utilization efficiency of the AWGs; it has been proven to reach $100 \%$ [25].

This device will be realized monolithically using PLC technologies. To confirm device feasibility, experiments using two separate uniform-loss and cyclic frequency (ULCF [70]) $32 \times 32$ AWGs connected with 32 optical fibers have been performed [24]. The device can support four input fibers simultaneously. Each fiber accommodates 32 100$\mathrm{GHz}$ spaced channels $(192.8+0.1 \times n \mathrm{THz} ; n=0-31)$ on an ITU-T grid. Each fiber carries four discontinuous bands. Each band consists of eight wavelength channels. One of the important characteristics of the MUX/DEMUX is crosstalk. The output WB configurations from the second AWG differ according to the input fiber connection patterns, which determine the magnitude of crosstalk level at each band. The crosstalk deteriorates when neighboring output ports carry the same WB from different input fibers or carry neighbor- ing WB on the same or different input fibers. The device allows different input fiber connection patterns and the patterns that minimize crosstalk have been identified and have been experimentally confirmed [24].

\subsection{Waveband Conversion Technologies}

Waveband conversion resolves waveband collision when routing wavebands and increases waveband accommodation efficiency within each fiber. The technology is much more difficult than single wavelength conversion. The requirements for waveband conversion are low crosstalk, small guard band, and bit rate and modulation format independency. Parametric wavelength conversion is one solution, however, it usually requires wide guard bands around the pump wavelength so as to minimize signal quality degradation due to the parametric crosstalk among the WDM signals. The degradation occurs in the conversion process and is enhanced when the channel spacing narrows. Conversion of $100 \mathrm{GHz}$-spaced 32 channels has been demonstrated with a $12.8 \mathrm{~nm}$ guard band, using fiber four wave mixing [71].

The performance has been improved by using periodically poled $\mathrm{LiNbO}_{3}$ (PPLN) waveguides based on quasiphase matching (QPM) [72], [73]. Due to the strict phase matching condition possible, the efficiency can be raised, and narrower-channel-spaced wavelength conversion with low crosstalk and small guard band be expected. Using a high efficiency PPLN waveguide, inter-band wavelength conversion of $25 \mathrm{GHz}$-spaced, $1.03 \mathrm{Tbit} / \mathrm{s}(103 \times$ $10 \mathrm{Gbit} / \mathrm{s}$ ) DWDM signals (C-band to L-band) has been reported [74]. The guard band was quite small at $8 \mathrm{~nm}$ $(4 \mathrm{~nm} \times 2)$ and converted signal Q-factors exceeded 15.6 dB. The technology was also used to create a polarizationindependent waveband converter that supported $25 \mathrm{GHz}$ spaced, $10 \mathrm{Gbit} / \mathrm{s} \times 64$ channels. It was coupled with an $8 \times 8$ PLC optical switch to demonstrate waveband path switching with a throughput of $5.12 \mathrm{Tbit} / \mathrm{s}$ across $32 \mathrm{~km}$ of field installed fibers in JGN-II (Japan Gigabit Network-II) testbed [75].

It has been shown that the two wavelength pump method can convert an arbitrary wavelength to another arbitrary wavelength [76], [77], and further, the simultaneous and arbitrary wavelength conversion of a waveband is possible [78] by using a LN waveguide having multiple QPM wavelengths [79], [80]. Waveband conversion technologies continue to advance, however, further improvements in terms of conversion efficiency and cost are required before their practical application can be considered.

\section{Conclusions}

This paper discussed the next step in photonic network evolution, the creation of hierarchical optical path networks. Key enabling technologies and recent advances were investigated. The introduction of wavebands was shown to reduce optical switch size at cross-connects, which mitigates one of the major barriers to the implementation of large throughput 
optical cross-connect systems. One of the other obstacles, network design complexity, was shown to be effectively resolved by a newly developed hierarchical optical path network design method that introduces a traffic demand expression in a Cartesian product space. Some of the key component technologies were also discussed and a new waveband MUX/DEMUX was demonstrated. As discussed in the paper, the hierarchical path arrangement is an efficient and effective means to create reliable large-throughput networks. The hierarchical optical path network will be implemented in the not so distant future when traffic volumes warrant it.

\section{Acknowledgments}

Part of this work was supported by NICT (National Institute of Information and Communications Technology) and JST (Japan Science and Technology Agency), to which we are deeply indebted. We are also grateful to Dr. Atushi Takada, NTT Network Innovation Laboratories, Mr. Isao Yagyu and Mr. Shoji Kakehashi, Nagoya University, for their useful discussions.

\section{References}

[1] K. Sato, S. Okamoto, and H. Hadama, "Optical path layer technologies to enhance B-ISDN integrity," Proc. ICC'93, pp.1300-1307, Geneva, May 1993.

[2] K. Sato, S. Okamoto, and H. Hadama, "Network integrity enhancement with optical path layer technologies," IEEE J. Sel. Areas Commun., vol.12, no.1, pp.159-170, Jan. 1994.

[3] K. Sato, S. Okamoto, and I. Tokizawa, Patent no.3221457 and 3247886, Optical Communications Network.

[4] I. Redpath, D. Cooperson, and R. Kline, "Metro WDM networks develop an edge," Proc. OFC/NFOFC 2006, NThC1, Anaheim, March 2006.

[5] G.K. Cambron, "The multimedia transformation," Presented at OFC '06, Plenary Presentation, Anaheim, March 2006.

[6] H. Tsushima and Y. Fukahiro, "OTN-based optical cross-connect systems to create reliable and transparent optical networks," Proc. SPIE, vol.6012, 601208-1-10, OpticsEast2005 (ITCom2005), Boston, Oct. 2005.

[7] S. Okamoto, et al., "Nationwide GMPLS field trial using different types (MPLS/TDM/Lambda) of switching capable equipment from multiple vendors," OFC 2005, Post deadline paper, PDP40, Anaheim, March 2005.

[8] L. Smarr, J. Ford, P. Papadopoulos, S. Fainman, T. DeFanti, M. Brown, and J. Leigh, "The OptIPuter, quartzite, and Starlight projects: A campus to global-scale testbed for optical technologies enabling LambdaGrid computing," OFC 2005, OWG7, Anaheim, March 2005.

[9] L. Gommans, "Generic AAA based optical networking," SURFnet Research on Networks Workshop, Utrecht, April 2004.

[10] M. Brown, ed., "Blueprint for the future of high-performance networking," Commun. ACM, vol.46, no.11, pp.30-77, Nov. 2003.

[11] G. Karmous-Edwards, "Today's optical network research infrastructures for Escience applications," Proc. OFC/NFOFC 2006, OWU3, Anaheim, March 2006.

[12] K. Harada, K. Shimizu, T. Kudou, and T. Ozeki, "Hierarchical optical path cross-connect systems for large scale WDM networks," Proc. OFC 1999, pp.356-358, Feb. 1999.

[13] L. Noirie, C. Blaizot, and E. Dotaro, "Multi-granularity optical cross-connect," Proc. Eur. Conf. Optical Communication (ECOC), pp.269-270, Oct. 2000.
[14] J. Yamawaku, A. Takada, W. Imajuku, and T. Morioka, "Evaluation of amount of equipment on single-layer optical paths," J. Lightwave Technol., vol.23, no.6, pp.1971-1978, June 2005.

[15] Y. Okano, T. Kawata, and T. Miki, "Designing digital paths in transmission networks," Proc. GLOBECOM 86, 25.2.1-25.2.5, Houston, 1986

[16] Y. Okano, S. Ohta, and T. Kawata, "Assessment of cross-connect systems in transmission networks," Proc. GLOBECOM 87, pp.1518, Tokyo, Japan, Nov. 1987.

[17] H. Kobrinski, "Crossconnection of wavelength-division-multiplexed high-speed channels," Electron. Lett., vol.23, no.18, pp.974-976, Aug. 1987.

[18] G. Hill, "Wavelength routing approach to optical communication networks," Br. Telecom Technical J., vol.6, no.3, pp.24-30, July 1988.

[19] G. Hill, P.J. Chidgy, and J. Davidson, "Wavelength routing for long haul networks," Proc. ICC'89, 23.3.1.-23.3.5, Boston, June 1989.

[20] X. Cao, V. Anand, and C. Qiao, "Waveband switching in optical networks," IEEE Commun. Mag., vol.41, no.4, pp.105-112, April 2003.

[21] I. Yagyu, H. Hasegawa, and K. Sato, "An efficient optical path network design algorithm based on a traffic demand expression in a Cartesian product space," Eur. Conf. Optical Communication (ECOC), Sept. 2006.

[22] Y. Suemura, I. Nishioka, Y. Maeno, S. Araki, R. Izmailov, and S. Ganguly, "Hierarchical routing in layered ring and mesh optical networks," ICC 2002, vol.5, pp.2727-2733, April-May 2002.

[23] R. Izmailov, S. Ganguly, V. Klepsyn, and A. Varsou, "Non-uniform waveband hierarchy in hybrid optical networks," Proc. IEEE INFOCOM'03, vol.2, pp.1344-1354, 2003.

[24] S. Kakehashi, H. Hasegawa, K. Sato, and O. Moriwaki, "Waveband MUX/DEMUX using concatenated arrayed-waveguide gratings," ECOC 2006, Cannu, Sept. 2006.

[25] S. Kakehashi, H. Hasegawa, K. Sato, and O. Moriwaki, "Formulation of waveguide connection for waveband MUX/DEMUX using concatenated arrayed-waveguide gratings," to appear in IEICE Trans. Commun.

[26] K. Sato, Advances in Transport Network Technologies -Photonic Networks, ATM, and SDH, Artech House, Norwood, MA, 1996. (ISBN 0-8906-851-8).

[27] ITU-T Recommendation G.803, Architecture of transport networks based on the synchronous digital hierarchy (SDH), March 2000.

[28] ITU-T Recommendation G.805, Generic functional architecture of transport networks, March 2000.

[29] M. Sexton and A. Reid, Broadband Networking: ATM, SDH, and SONET, Artech House, Norwood, MA, 1997 (ISBN 0-89006-578$0)$.

[30] K. Sato, S. Ohta, and I. Tokizawa, "Broadband transport network architecture based on virtual paths," IEEE Trans. Commun., vol.38, no.8, pp.1212-1222, Aug. 1990.

[31] K. Sato and I. Tokizawa, "Flexible asynchronous transfer mode networks utilizing virtual paths," Proc. ICC'90, 318.4.1.-318.4.8., Atlanta, USA, April 1990.

[32] K. Sato, et al., Packet Multiplexed Communication Systems, Patent No.2670613 and 2825156 .

[33] "Multiprotocol label switching architecture," IETF RFC 3031, Jan 2001.

[34] B. Davie and Y. Rekhter, MPLS: Technology and Applications, Morgan Kaufmann, 2000 (ISBN 1-55860-656-4).

[35] E. Mannie, ed., Generalized Multi-Protocol Label Switching (GMPLS) Architecture, IETF REC3945, Oct. 2004.

[36] N. Yamanaka, K. Shiomoto, and E. Oki, eds., GMPLS Technologies-Broadband Backbone Networks and Systems, CRC Press, 2005 (ISBN: 0-8247-2781-9).

[37] B.W. Stuck, "The digital cross-connect: Cornerstones of future networks?," Data Communications, pp.165-178, Aug. 1987.

[38] A. Gladisch, R.-P. Braun, D. Breuer, A. Ehrhardt, H.-M. Foisel, M. 
Jaeger, R. Leppla, M. Schneiders, S. Vorbeck, W. Weiershausen, and F.-J. Westphal, "Evolution of terrestrial optical system and core network architecture," Proc. IEEE, vol.94, no.5, pp.869-891, May 2006.

[39] K. Sato, "Recent developments in and challenges of photonic networking technologies," IEICE Trans. Commun., vol.E90-B, no.3, pp.454-467, March 2007.

[40] I. Chlamtac, A. Ganz, and G. Karmi, "Lightpath communications: An approach to high bandwidth optical WAN's," IEEE Trans. Commun., vol.40, no.7, pp.1171-1182, July 1992.

[41] P.-H. Ho, H.T. Mouftah, and J. Wu, "A scalable design of multigranularity optical cross-connects for the next- generation optical Internet," IEEE J. Sel. Areas Commun., vol.21, no.7, pp.1133-1142, Sept. 2003.

[42] A. Kolarov, T. Wang, B. Sengupta, and M. Cvijetic, "Impact of waveband switching on dimensioning multi-granular hybrid optical networks," Proc. Conference on Optical Network Design and Modeling 2005, pp.371-381, Feb. 2005.

[43] Y. Shun, C. Ou, and B. Mukherjee, "Design of hybrid optical networks with waveband and electrical TDM switching," Proc. IEEE Global Telecommunications Conference 2003 (GLOBECOM'03) vol.5, pp.2803-2808, Dec. 2003.

[44] S.S.W. Lee, M.C. Yuang, and P.-L. Tien, "A Lagrangean relaxation approach to routing and wavelength assignment for multigranularity optical WDM networks," Proc. IEEE Global Telecommunications Conference 2004 (GLOBECOM'04), vol.3, pp.19361942, Nov. 2004.

[45] P.-H. Ho and H.T. Mouftah, "Routing and wavelength assignment with multigranularity traffic in optical networks," IEEE J. Lightwave Technol., vol.20, no.8, pp.1292-1303, Aug. 2002.

[46] M. Lee, J. Yu, Y. Kim, C. Kang, and J. Park, "Design of hierarchical crossconnect WDM networks employing a two-stage multiplexing scheme of waveband and wavelength," IEEE J. Sel. Areas Commun., vol.20, no.1, pp.166-171, Jan. 2002.

[47] X. Cao, V. Anand, Y. Xiong, and C. Qiao, "A study of waveband switching with multilayer multigranular optical crossconnects," IEEE J. Sel. Areas Commun., vol.21, no.7, pp.1081-1094, Sept. 2003

[48] I. Yagyu, H. Hasegawa, and K. Sato, "An efficient waveband path assignment algorithm for hierarchical optical path network based on a traffic demand expression in a cartesian product space," IEICE Technical Report, PN 2006-12, Aug. 2006.

[49] K. Zhu and B. Mukherjee, "A review of traffic grooming in WDM optical networks: Architectures and challenges," Optical Network Magazine, vol.4, no.2, pp.55-64, March/April 2003.

[50] W. Yao and B. Ramamurthy, "Survivable traffic grooming with path protection at the connection level in WDM mesh networks," IEEE/OSA J. Lightwave Technol., vol.23, no.10, pp.2846-2853, Oct. 2005.

[51] M. Kodialam and T.V. Lakshman, "Dynamic routing of locally restorable bandwidth guaranteed tunnels using aggregated link usage information," Proc. IEEE INFOCOM 2001, pp.376-385, April 2001

[52] H. Zhu, H. Zang, K. Zhu, and B. Mukherjee, "A novel generic graph model for traffic grooming in heterogeneous WDM mesh networks," IEEE/ACM Trans. Netw., vol.11, no.2, pp.285-299, April 2003.

[53] J. Yamawaku, A. Takada, W. Imajuku, and T. Morioka, "Evaluation of amount of equipment on single-layer optical paths," J. Lightwave Technol., vol.23, no.6, pp.1971-1978, June 2005.

[54] L.Y. Lin, E.L. Goldstein, J.M. Simmons, and R.W. Tkach, "Freespace micromachined optical switches with submillisecond switching time for large-scale optical crossconnects," IEEE Photonics Technol. Lett., vol.10, no.4, pp.525-527, 1998.

[55] V. Kaman, R. Anderson, R. Helkey, O. Jerphagnon, A. Keating, B Liu, H. Poulsen, C. Pasarla, D. Xu, S. Yuan, and X. Zheng, "Optical performance of a $288 \times 288$ photonic cross-connect system," Proc. of PS2002, paper-PS. TuA4, pp.59-61, 2002.
[56] M. Okuno, A. Sugita, T. Matsunaga, M. Kawachi, Y. Ohmori, and K Katoh, " $8 \times 8$ optical matrix switch using silica-based planar lightwave circuit," IEICE Trans. Electron., vol.E76-C, no.7, pp.12151223, July 1993.

[57] M. Yano, F. Yamagishi, and T. Tsuda, "Optical MEMS for photonic switching-compact and stable optical crossconnect switches for simple, fast, and flexible wavelength applications in recent photonic networks," IEEE J. Sel. Top. Quantum Electron., vol.11, no.2, pp.383394, March/April, 2005.

[58] D.M. Marom, D.T. Neilson, D.S. Greywall, N.R. Basavanhally, P.R Kolodner, Y.L. Low, F. Pardo, C.A. Bolle, S. Chandrasekhar, L. Buhl, C.R. Giles, S.-H. Oh, C.S. Pai, K. Werder, H.T. Soh, G.R Bogard, E. Ferry, F.P. Klemens, K. Teffeau, J.F. Miner, S. Rogers, H.E. Bower, R.C. Keller, and W. Manfield, "Wavelength-selective $1 \times 4$ switch for $128 \mathrm{WDM}$ channels at $50 \mathrm{GHz}$ spacing," OFC 2002 , Postdeadline Papers, FB7-1-FB7-3, Anaheim, CA, March 2002.

[59] J. Tsai, L. Fan, C. Chi, D. Hah, and M.C. Wu, "A large-count $1 \times$ 32 wavelength-selective switch using a large scan-angle, high fillfactor, two-axis analog micromirror array," ECOC 2004, Tu1.5.2, Stockholm, Sept. 2004.

[60] S. Okamoto, A. Watanabe, and K. Sato, "A new optical path crossconnect system architecture utilizing delivery and coupling matrix switch,” IEICE Trans. Commun., vol.E77-B, no.10, pp.1272-1274, Oct. 1994.

[61] A. Watanabe, S. Okamoto, and K. Sato, "Optical switch using WDM," Patent no.3416895.

[62] A. Watanabe, S. Okamoto, K. Sato, and M. Okuno, "Optical switch," Patent no.3444548.

[63] M. Okuno, T. Goh, S. Sohma, and T. Shibata, "Recent advances in optical switches using silica-based PLC technology," NTT Technical Review, vol.1, no.7, pp.20-30, Oct. 2003.

[64] C. Clos, "A study of non-blocking switching networks," Bell Syst. Tech. J., vol.32, no.2, pp.406-424, 1953.

[65] S. Kakehashi, H. Hasegawa, and K. Sato, "Optical switch architectures for hierarchical optical path networks," OpticsEast 2006 , Boston, MA, Oct. 2006.

[66] G.J. Ockenfuss, "Ultra-low stress coating process: An enabling technology for extreme performance thin film interference filters," Proc. OFC, Postdeadline Paper, FA8-1-FA8-4, 2002.

[67] C.R. Doerr, R. Pafchek, and L.W. Stulz, "Integer band demultiplexer using waveguide grating routers," IEEE Photonics Technol. Lett., vol.15, no.8, pp.1088-1090, Aug. 2003.

[68] S. Chandrasekhar, C.R. Doerr, and L.L. Buhl, "Flexible waveband optical networking without guard bands using novel 8-skip-0 banding filters," IEEE Photonics Technol. Lett., vol.17, no.3, pp.579581, March 2005.

[69] C. Dragone, C.A. Edwards, and R.C. Kistler, "Integrated optics $N \times N$ multiplexer on silicon," IEEE Photonics Technol. Lett., vol.3, no.10, pp.896-899, Oct. 1991.

[70] K. Okamoto, T. Hasegawa, O. Ishida, A. Himeno, and Y. Ohmori, "32 × 32 arrayed-waveguide grating multiplexer with uniform loss and cyclic frequency characteristics," Electron. Lett., vol.33, pp.1865-1866, 1997

[71] S. Watanabe, S. Takeda, and T. Chikama, "Interband wavelength conversion of $320 \mathrm{Gbit} / \mathrm{s}(32 \times 10 \mathrm{Gbit} / \mathrm{s})$ WDM signal using a polarization-insensitive fiber four-wave mixer," ECOC'98, Postdeadline paper, pp.85-86, 1998.

[72] M.H. Chou, I. Brener, M.M. Fejer, E.E. Chaban, and S.B Christman, "1.5- $\mu$-band wavelength conversion based on cascaded second-order nonlinearity in $\mathrm{LiNbO}$ waveguides," IEEE Photonics Technol. Lett., vol.11, no.6, pp.653-655, June 1999.

[73] O. Tadanaga, M. Asobe, H. Miyazawa, Y. Nishida, and H. Suzuki, "A 1-THz optical frequency shifter using quasi-phase-matched$\mathrm{LiNbO}_{3}$ wavelength converters," Proc. OFC, ThDD1, pp.592-593, March 2002.

[74] J. Yamawaku, H. Takara, T. Ohara, K. Sato, A. Takada, T. Morioka, O. Tadanaga, H. Miyazawa, and M. Asobe, "Inter-band wavelength 
conversion of $25 \mathrm{GHz}$-spaced $1.03 \mathrm{Tbit} / \mathrm{s}(103 \times 10 \mathrm{Gbit} / \mathrm{s}) \mathrm{DWDM}$ signals with small guard band and low crosstalk in PPLN waveguide," Proc. CLEO 2003, paper CThPDB2, Baltimore, June 2003.

[75] J. Yamawaku, E. Yamazaki, A. Takada, T. Morioka, and K. Suzuki, "Virtual grouped-wavelength-path switching based on QPM-LN waveband converter and supercontinuum wavelength-bank source," Proc. OFC2005, paper OFE2, Anaheim, March 2005.

[76] R. Osellame, R. Ramponi, M. Marangoni, G. Tartarini, and P. Bassi, "Integrated all-optical nonlinear device for reconfigurable add/drop and wavelength shifting of WDM signals," Appl. Phys. B, vol.73, no.5-6, p.505, 2001.

[77] J. Yamawaku, A. Takada, E. Yamazaki, O. Tadanaga, H. Miyazawa, and M. Asobe, "Selective wavelength conversion using PPLN waveguide with two pump configuration," CLEO 2003, CWB5, Baltimore, 2003.

[78] E. Yamazaki, A. Takada, J. Yamawaku, and T. Morioka, "Simultaneous and arbitrary wavelength conversion of WDM signals using multiple wavelength quasi phase matched $\mathrm{LiNbO}_{3}$ waveguide," Proc. OFC 2004, paper FL6, Los Angels, March 2004.

[79] M.H. Chou, K.R. Parameswaran, M.M. Fejer, and I. Brener, "Multiple-channel wavelength conversion by use of engineered quasi-phase-matching structures in $\mathrm{LiNbO}_{3}$ waveguides," Opt. Lett., vol.24, no.16, pp.1157-1159, Aug. 1999.

[80] M. Asobe. O. Tadanaga, H. Miyazawa, Y. Nishida, and H. Suzuki, "Highly flexible and robust multiple quasi-phase matched $\mathrm{LiNbO}_{3}$ wavelength converter," CLEO 2003, paper CWB3, pp.1129-1132, Baltimore, 2003.

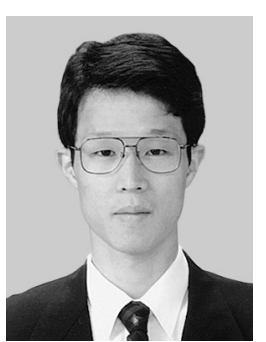

Hiroshi Hasegawa received the B.E., M.E., and D.E. degrees all in Electrical and Electronic Engineering from Tokyo Institute of Technology, Tokyo, Japan, in 1995, 1997, and 2000, respectively. From 2000 to 2005, he was a research associate of Dept. of Communications and Integrated Systems, Tokyo Institute of Technology. Currently he is an associate professor of Nagoya University. His current research interests include Photonic Networks, Image Processing (especially Superresolution), Multidimensional Digital Signal Processing and Time-Frequency Analysis. He received the Young Researcher's Awards from SITA (Society of Information Theory and its Applications) and IEICE (Institute of Electronics, Information and Communication Engineers) in 2003 and 2005, respectively. Dr. Hasegawa is a member of SITA and IEEE.

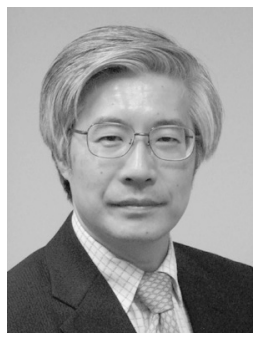

Ken-ichi Sato received the B.S., M.S., and $\mathrm{Ph} . \mathrm{D}$. degrees in Electronics Engineering from the University of Tokyo, in 1976, 1978, and 1986, respectively. He is currently a professor at the Graduate School of Engineering, Nagoya University, and he is an NTT R\&D Fellow. Before joining the university in April 2004, he was an executive manager of the Photonic Transport Network Laboratory at NTT. His R\&D activities cover future transport network architectures, network design, OA\&M (operation administration and maintenance) systems, photonic network systems including optical cross-connect/ADM and photonic IP routers, and optical transmission technologies. He has authored/co-authored more than 200 research publications in international journals and conferences. He holds 35 granted patents and more than 100 pending patents. He received the Young Engineer Award in 1984, the Excellent Paper Award in 1991, and the Achievement Award in 2000 from the Institute of Electronics, Information and Communication Engineers (IEICE) of JAPAN. He was also the recipient of the distinguished achievement Award of the Ministry of Education, Science and Culture in 2002. His contributions to ATM (Asynchronous Transfer Mode) and optical network technology development extend to co-editing three IEEE JSAC special issues and the IEEE JLT special issue once, organizing several Workshops and Conference technical sessions, serving on numerous committees of international conferences including OFC and ECOC, authoring a book, Advances in Transport Network Technologies (Artech House, 1996), and co-authoring thirteen other books. He is a Fellow of the IEEE. 